\title{
Recomendaciones sobre el diagnóstico y el tratamiento de la alergia a la proteína de la leche de vaca en población pediátrica colombiana - Posición de expertos
}

\section{Expert recommendations for diagnosis and treatment of cow's milk protein allergy in the Colombian pediatric population}

\author{
María Catalina Bagés M., MD, ${ }^{*}$ Carlos Fernando Chinchilla M., MD, ${ }^{2}$ Catalina Ortiz P., MD, ${ }^{3}$ Clara Eugenia Plata G., MD, ${ }^{4}$ \\ Enilda Martha Puello M., MD, ${ }^{5}$ Óscar Javier Quintero H., MD, ${ }^{6}$ Juan Pablo Riveros L., MD, ${ }^{7}$ Francisco Javier Sosa G., MD, ${ }^{8}$ \\ Alejandra Wilches L., MD, ${ }^{9}$ José Fernando Vera-Chamorro, MD. ${ }^{10}$
}

\footnotetext{
1. Pediatra y nutrióloga pediatra. Facultad de Medicina, Universidad El Bosque, Instituto de Investigación en Nutrición, Genética y Metabolismo, Bogotá, Colombia

2 Pediatra alergólogo clínico. Profesor en la Facultad de Medicina, Universidad de Antioquia, Medellín, Colombia.

${ }^{3}$ Gastroenteróloga infantil. Gastroenterología, hepatología y nutrición Pediátrica, Hospital Pablo Tobón Uribe, Medellín, Colombia.

${ }^{4}$ Gastroenteróloga pediatra, Hospital San Ignacio de Bogotá, Colombia.

5 Pediatra, máster en Nutrición Infantil. Clínica Iberoamericana de Barranquilla, Colombia.

${ }^{6}$ Gastroenterólogo pediatra y nutriólogo. Máster en Epidemiología. Profesor asociado en la Universidad del Rosario, Bogotá, Colombia.

7 Gastroenterólogo pediatra. Universidad del Bosque. Gastricare S. A. S., Gerente general, Bogotá, Colombia

${ }_{8}$ Gastroenterólogo pediatra. Sura EPS, Clínica Esimed de Medellín. Pediatra de cuidado intensivo neonatal. Bogotá, Colombia.

9 Gastroenteróloga pediatra, Hospital San Vicente Fundación. Medellín, Colombia.

${ }^{10}$ Gastroenterólogo pediatra. Máster en Ciencias de la Nutrición. Epidemiólogo clínico, Hospital Universitario Fundación Santa Fe de Bogotá; Universidad de los Andes, grupo de investigación: PediAFe. Bogotá, Colombia.

Coordinación Editorial: Integralis HGS (Doctores Daniel Rodríguez y María Stella Salazar)

*Correspondencia: María Catalina Bagés M., bagesmaria@unbosque.edu.co
}

Fecha recibido: $\quad 13 / 05 / 19$ Fecha aceptado: 24/09/19

\begin{abstract}
Resumen
Objetivo: generar recomendaciones sobre el diagnóstico y el tratamiento de la alergia a la proteína de la leche de vaca (APLV), que sirvan de referencia y consulta para los médicos pediatras y de cuidado primario. Materiales y métodos: el presente documento de posición de expertos fue desarrollado por un grupo de médicos, especialistas en diferentes áreas terapéuticas y con experiencia en APLV. Se definieron los temas más relevantes y se realizó una revisión de la literatura científica disponible, a fin de elaborar una propuesta de recomendaciones que fue discutida por los autores. Resultados: se elaboró un documento de posición que propone un enfoque práctico sobre la definición, el diagnóstico y el tratamiento de la APLV en el paciente pediátrico. Conclusiones: el diagnóstico temprano y el manejo adecuado de la APLV pueden contribuir a una disminución de la carga de esta enfermedad y sus complicaciones.
\end{abstract}

\section{Palabras clave}

Alergia a la proteína de la leche de vaca (APLV), atopia, lactancia materna, fórmulas para lactantes, fórmulas hidrolizadas, dermatitis atópica, lactantes.

\begin{abstract}
Objective: The objective of this paper is to develop and present recommendations for diagnosis and treatment of Cow's Milk Protein Allergy (CMPA) which can serve as a reference for pediatric and primary care physicians to consult. Materials and methods: This expert position document was developed by a group of doctors who are specialists in several therapeutic areas who have experience in CMPA. The most relevant topics were defined and a review of the available scientific literature was carried out to prepare a proposal for recommendations that was then discussed by the authors. Results: A position paper was developed that proposes a practical approach to definition, diagnosis and treatment of CMPA in pediatric patients. Conclusions: Early diagnosis and proper management of CMPA can help decrease the burden of this disease and its complications.
\end{abstract}

\section{Keywords}

Cow's milk protein allergy (CMPA), atopy, breastfeeding, infant formulas, hydrolyzed formulas, atopic dermatitis, infants.

\section{INTRODUCCIÓN}

La alergia a la proteína de la leche de vaca (APLV) es una condición clínica de origen inmunológico, que inicia a temprana edad y constituye la forma de alergia alimentaria más frecuente en los primeros meses de vida, incluso en niños de edad preescolar. Durante el primer año de vida, la proteína de la leche de vaca (PLV) suele ser el primer antí- 
geno al cual se enfrentan los niños alimentados con leche materna o con fórmulas para lactantes $(1,2)$.

Así, la APLV se ha convertido en un importante problema de salud, debido a sus manifestaciones clínicas y a sus consecuencias. Es probable que la APLV pueda estar asociada al desarrollo de otro tipo de alergias como asma, dermatitis atópica y rinitis $(1,2)$.

La información sobre la incidencia y la prevalencia de la APLV es difícil de establecer, validar y comparar, dados los múltiples factores como un reducido número de estudios epidemiológicos, las deficiencias o inconsistencias en el diseño de los estudios, la falta de estandarización en el método de diagnóstico (autorreporte, prueba de reto, niveles de inmunoglobulina $\mathrm{E}$ [IgE] específica, prueba de punción, entre otros) y datos reportados principalmente con relación a las reacciones rápidas (se desconoce lo acontecido con reacciones de hipersensibilidad tardía).

Además, gran parte de los datos disponibles se relacionan con alergia a otros alimentos más que a la PLV. En efecto, los reportes resultantes de encuestas (autorreporte) pueden corresponder a diagnósticos errados (intolerancia) o a niños a quienes no les gustan ciertos alimentos, $y$, finalmente, las manifestaciones de la APLV no necesariamente son gastrointestinales.

De acuerdo con el estudio europeo EuroPrevall, la incidencia de la APLV es de 1-4 \%, pero disminuye al 1-2\% cuando se hacen estudios más específicos (3). En Estados Unidos de América, se publicaron revisiones en 2007 y 2010 en las cuales se reporta una prevalencia de APLV del $3 \%$, según una encuesta. Dichos resultados no corresponden con los de estudios específicos como la prueba de punción o el reto a la PLV, en los que la prevalencia ha oscilado entre el 0,6 y el $1 \%$. En general, la prevalencia podría ser, aproximadamente, del $1 \%(4,5)$.

Entre tanto, los reportes específicos de la APLV en Latinoamérica son escasos. De acuerdo con cifras reportadas por Ávila Castañón y colaboradores, la prevalencia de alergia a la leche (prueba de punción) en pacientes del Hospital Infantil de México es de 7,7 \%. En Chile, Martínez reportó cifras de prevalencia de APLV que oscilaron entre el 2,5 y el $7,1 \%$ (prueba de punción). Se debe considerar que la prevalencia depende del tipo de estudio realizado (encuesta, prueba de punción, IgE específica, prueba de radioalergoabsorbencia [Radio Allergo Sorbent Test, RAST]) $(6,7)$.

La información epidemiológica sobre la APLV en Colombia es escasa. De acuerdo con los resultados publicados por Marrugo (encuesta), la prevalencia de la APLV en niños entre 1 y 8 años es del 2,5\%, mientras que entre los 9 y 16 años es del 11,1\% (8). Según la publicación de Acevedo, la prevalencia de APLV en niños $<2$ años es del $1,8 \%$ (9). Los resultados de estos estudios, sin embargo, no se pueden considerar concluyentes en cuanto a su representatividad de la población colombiana en general (8-12).

Las manifestaciones gastrointestinales de la APLV no son específicas y pueden confundirse con las de la enfermedad por reflujo gastroesofágico (ERGE), los trastornos gastrointestinales funcionales, la intolerancia a la lactosa o las reacciones a alimentos de origen no alérgico. Este escenario da como consecuencia una alta probabilidad de sobre o subdiagnóstico.

En consecuencia, es un reto para el médico hacer un diagnóstico temprano y correcto de la APLV, así como establecer un manejo oportuno y apropiado de la enfermedad, con el fin de mejorar el pronóstico de los pacientes y de minimizar la carga de esta. En respuesta a estas situaciones, un grupo de médicos con experiencia y conocimiento en el campo de la APLV decidieron reunirse y desarrollar un documento de posición. Con ello apuntan a proponer un enfoque práctico sobre la definición, el diagnóstico y el tratamiento de la APLV en el paciente pediátrico.

\section{MATERIALES Y MÉTODOS}

Las recomendaciones de este documento se basan en la revisión de la literatura científica de mejor calidad disponible sobre la APLV y en la experiencia de un grupo de médicos especialistas en diversas áreas de la pediatría (alergólogos, gastroenterólogos, nutriólogos y epidemiólogos clínicos), procedentes de diferentes regiones de Colombia. Posterior a la revisión de la literatura, se realizó una reunión para discutir las recomendaciones hasta lograr un acuerdo.

\section{RECOMENDACIONES}

\section{Definiciones}

- Atopia: es la predisposición genética del individuo a producir cantidades elevadas de $\mathrm{IgE}$ ante antígenos específicos $(1,13)$.

- Intolerancia alimentaria: es una reacción adversa a un alimento, no relacionada con un mecanismo inmunológico. Un ejemplo de ello es la intolerancia a la lactosa, que no es mediada inmunológicamente, sino provocada por la deficiencia funcional de la enzima lactasa $(\beta$-galactosidasa), que ocasiona la absorción incompleta de la lactosa $(1,13)$.

- Alergia: es una reacción de hipersensibilidad mediada por mecanismos inmunológicos específicos. En la mayoría de los niños, esta condición es mediada por la $\operatorname{IgE}$, con la expresión fenotípica de atopia, con o sin eccema atópico, rinitis alérgica o asma. Otros niños presentan manifestaciones gastrointestinales no mediadas 
por la $\operatorname{IgE}$ (mediadas por células), frecuentes en los síntomas de la enfermedad (14).

- Alergia a la proteína de la leche de vaca (APLV): es una reacción de hipersensibilidad mediada por mecanismos inmunológicos específicos y reproducibles, que causa manifestaciones clínicas adversas. La reacción inmune puede ser mediada por la IgE, no mediada por la IgE o mixta. Se trata de una alergia a epítopos de origen proteico $(1,13,14)$.

\section{DIAGNÓSTICO CLÍNICO}

\section{Factores de riesgo}

Entre los factores de riesgo asociados con la aparición de la APLV se incluyen madre fumadora, padres y hermanos atópicos; nacimiento por cesárea; inicio de fórmula láctea en los primeros días de vida; lactancia por menos de 3 meses; madre con más de 30 años; prematuridad, e hiperbilirrubinemia $(1,15-20)$ (Tabla 1).

Tabla 1. Factores por considerar en pacientes con sospecha de APLV

\section{Edad de inicio.}

Naturaleza de los síntomas

Frecuencia de las manifestaciones

Tiempo entre la ingesta y el inicio de los síntomas.

Cantidad de leche necesaria para provocar los síntomas.

Método de preparación de la leche.

Reproducibilidad de la reacción.

Intervalo de tiempo desde la última reacción.

Diario de alimentación.

Influencia de factores externos sobre la reacción (ejemplo: ejercicio, cambios hormonales, estrés).

Evaluación antropométrica con progreso pondoestatural.

Detalles tempranos de alimentación (duración de lactancia materna, uso de sucedáneos, ablactación).

Efecto de las dietas de eliminación (soya, fórmulas terapéuticas, dieta materna durante lactancia).

Intervenciones terapéuticas.

Modificada de la referencia 14

\section{Manifestaciones clínicas}

Las manifestaciones clínicas de la APLV se pueden presentar en cualquier edad. Por tanto, los siguientes factores deben ser investigados en pacientes con sospecha de $\operatorname{APLV}(1,14,21)$ :

\section{Síntomas inmediatos mediados por lgE}

Son síntomas cuya severidad puede ir de leve a grave y que se desarrollan en los minutos siguientes a la exposi- ción al alérgeno. Además, comprometen la piel (urticaria, angioedema), el tracto gastrointestinal (vómito, enterocolitis), el sistema respiratorio (sibilancias, estridor, disnea) y el sistema cardiovascular (Tabla 2). En lactantes pequeños, los síntomas pueden ser inespecíficos, como palidez y adinamia (22-24).

Tabla 2. Otros síntomas inmediatos mediados por $\operatorname{IgE}$

\begin{tabular}{|c|c|}
\hline Cutáneos & $\begin{array}{l}\text { Prurito sin lesiones de piel, urticaria, } \\
\text { angioedema, exacerbación del eccema atópico. }\end{array}$ \\
\hline Gastrointestinales & $\begin{array}{l}\text { Vómito, diarrea, rectorragia, reflujo } \\
\text { gastroesofágico, dolor abdominal. }\end{array}$ \\
\hline Respiratorios & $\begin{array}{l}\text { Rinitis, congestión nasal, sibilancias, tos, } \\
\text { estridor, dificultad respiratoria. }\end{array}$ \\
\hline Cardiovasculares & Hipotensión/choque. \\
\hline Generales & $\begin{array}{l}\text { Hipotonía, anafilaxia, postración, irritabilidad, } \\
\text { falla de crecimiento. }\end{array}$ \\
\hline
\end{tabular}

Modificada de la referencia 24

Asimismo, la anafilaxia es uno de los síntomas inmediatos y se define como una reacción alérgica grave sistémica o generalizada. Esta se constituye en la manifestación más crítica de la APLV inmediata, la cual causa una mortalidad cercana al $10 \%(1,25-27)$.

\section{Síntomas tardíos no mediados por IgE o mixtos}

Se presentan varias horas o días después de la ingesta del alérgeno (Tabla 3). Estos síntomas comprometen predominantemente el tracto gastrointestinal, pero casi siempre son múltiples $(2,14,19,28,29)$.

Tabla 3. Síntomas tardíos no mediados por IgE o mixtos

\begin{tabular}{|c|c|}
\hline $\begin{array}{l}\text { Manifestaciones } \\
\text { cutáneas }\end{array}$ & Dermatitis atópica, urticaria, angioedema. \\
\hline $\begin{array}{l}\text { Manifestaciones } \\
\text { gastrointestinales }\end{array}$ & $\begin{array}{l}\text { Náuseas, vómito, anorexia, disfagia, } \\
\text { regurgitación, cólico, anorexia, diarrea, } \\
\text { estreñimiento, sangre en heces, impactación } \\
\text { fecal, reflujo gastroesofágico. }\end{array}$ \\
\hline $\begin{array}{l}\text { Manifestaciones } \\
\text { respiratorias }\end{array}$ & Rinorrea, sibilancia, tos crónica. \\
\hline Generales & Anafilaxia. \\
\hline
\end{tabular}

Modificada de las referencias 2, 14, 19, 28 y 29

Entre los síntomas gastrointestinales tardíos más comunes se pueden mencionar vómito, irritabilidad (cólico), disfagia, diarrea, estreñimiento, falla del crecimiento y rectorragia.

Además de la sintomatología, el diagnóstico clínico puede hacerse por medio de la eliminación de alimentos (leche y sus derivados o cualquier alimento que contenga ingredientes lácteos) y provocación (protocolos de reto/ 
desafío alimentario, doble ciego controlado con placebo, que son considerados el tratamiento de referencia). Así pues, el tratamiento de referencia del diagnóstico clínico es la eliminación de la PLV durante 2-4 semanas y la reintroducción de la PLV en forma de reto/desafío abierto o reto/ desafío ciego (reto/desafío diagnóstico) $(18,20)$.

\section{Diagnóstico diferencial}

Es importante considerar otras entidades que podrían confundirse con APLV, como la intolerancia a la lactosa, la acidosis tubular renal, la hipertrofia pilórica, la fibrosis quística o enterocolitis, la enfermedad por reflujo gastroesofágico, entre otras condiciones.

\section{Recomendaciones}

- Es fundamental elaborar una historia clínica que incluya la determinación de factores de riesgo para APLV y que permita establecer un diagnóstico temprano.

- Antes de diagnosticar una nueva alergia o una alergia múltiple, se debe pensar que el paciente puede estar expuesto a la ingesta de derivados ocultos de la leche o a trazas en los alimentos.

- La mayoría de los síntomas alérgicos inducidos por la leche se desarrollan en minutos u horas después de la ingesta, excepto en las alergias gastrointestinales.

- La APLV, clásicamente, presenta signos y síntomas en la piel, el tracto gastrointestinal y el tracto respiratorio.

- Se debe realizar un diagnóstico diferencial de la APLV.

- Los cambios de la microbiota intestinal son responsables, en parte, del aumento de la presencia de enfermedades alérgicas. Lo ideal sería entonces mejorar la microbiota intestinal desde el inicio (procurando un parto vaginal, el inicio de leche materna en la primera hora de vida y evitando el uso indiscriminado de antibióticos), con el fin de disminuir el riesgo de aparición de estos cuadros $(30,31)$.

\section{DIAGNÓSTICO: LABORATORIO CLÍNICO Y PRUEBAS INMUNOLÓGICAS}

El manejo de cada paciente debe ser individualizado, a partir de la realización de diferentes recursos diagnósticos.

\section{Pruebas diagnósticas}

- Prueba de provocación oral alimentaria: el tratamiento de referencia para el diagnóstico de la APLV es la prueba de exposición que debe ser realizada por el médico especialista (gastroenterólogo o alergólogo). No se recomienda efectuar pruebas de reto/desafío, si no se dispone de la experiencia y el conocimiento necesarios para garantizar la seguridad del paciente. Las pruebas de provocación oral con leche de vaca son diagnósticas in vivo, desplegadas para confirmar definitivamente una sospecha preliminar de la APLV.

Una prueba que deriva en una reacción clínica se define como positiva o provocación fallida, mientras que una sin reacción clínica se denomina provocación negativa o aprobada. Así, una provocación positiva indicará la dosis tolerada, si la hay, y permitirá la planificación de dietas de exclusión parcial o completa de $\operatorname{PLV}(14,18,20)$.

- Exámenes paraclínicos básicos: cuadro hemático, frotis de sangre periférica, ferritina, proteínas totales y diferenciales.

- Valoración nutricional: todo paciente requiere una evaluación nutricional completa para detectar el riesgo nutricional de ingesta de alérgenos.

- Prueba intraepidérmica: tiene una alta sensibilidad, así como un alto valor predictivo negativo, pero baja especificidad. Confirma la sensibilización, pero no es diagnóstica de APLV.

- Niveles de IgE específicos: se ha establecido, específicamente, para leche de vaca un punto de corte $>5 \mathrm{KU} / \mathrm{L}$ en $<1$ año y $>15 \mathrm{KU} / \mathrm{L}$ en $>1$ año, con probabilidad del $95 \%$ en una prueba de exposición oral positiva (14).

- Prueba dérmica de sensibilización: no se recomienda su uso en pacientes con una alergia alimentaria especialmente mediada por la IgE (14).

\section{Recomendaciones}

La mayoría de los pacientes vistos en la consulta pediátrica presentan procesos no mediados por la $\operatorname{IgE}$ y no requieren de pruebas inmunológicas diagnósticas.

- En pacientes con sospecha de APLV, con cuadro clínico compatible de reacciones mediadas por la IgE o mixtas, $y$ en pacientes con manifestaciones no mediadas por la $\mathrm{IgE}$, pero que no responden al tratamiento, se solicitan las siguientes pruebas: IgE específica (prueba RAST $o$ ImmunoCAP) y prueba de punción para leche de vaca. Luego de ello, se remite el paciente al especialista (gastroenterólogo o alergólogo pediatra).

- No se recomienda evaluar la IgE sérica total en estos pacientes.

- Las reacciones tardías no tienen pruebas diagnósticas convencionales. El diagnóstico es clínico por exclusión y por provocación.

- Si un paciente presenta una respuesta negativa a las pruebas inmunológicas, esto no descarta un proceso no mediado por la IgE. 


\section{DIAGNÓSTICO POR ENDOSCOPIA DIGESTIVA}

El principal factor que se debe considerar para solicitar una evaluación endoscópica del paciente con APLV es su sintomatología (independientemente de la edad) y la sospecha de esofagitis eosinofílica, o los pacientes con proctocolitis alérgica que presentan anemia y que persisten con manifestaciones no sólo de sangrado sino distensión abdominal y vómito persistente, las cuales pueden tener un componente mixto (32-34).

\section{Indicaciones de la endoscopia digestiva alta y baja en APLV}

La realización de pruebas endoscópicas en un paciente con diagnóstico de APLV no es una práctica común en este grupo de enfermedades, dado que el diagnóstico es clínico. Por tanto, la decisión de efectuar estas pruebas se basa en criterios clínicos bien definidos, según las características del paciente, su estado nutricional, pruebas de laboratorio y las manifestaciones clínicas evidentes. Las indicaciones de endoscopia digestiva se pueden observar en la Tabla $4(35,36)$.

Tabla 4. Indicaciones de la endoscopia digestiva

Signos de malabsorción, deficiencia de micro o macronutrientes y poca ganancia de peso o desnutrición, así como sospecha de gastroenteropatía eosinofílica.

Anemia e hipoalbuminemia en pacientes alérgicos.

Dolor abdominal persistente, distensión e hiporexia.

Inapetencia persistente y sospecha de esofagitis eosinofílica.

Hemorragia del tracto digestivo alto o bajo.

Diagnóstico de FPIES.

Sospecha de enfermedad inflamatoria intestinal.

Control durante el tratamiento o el postratamiento (p. ej. esofagitis eosinofílica).

FPIES: síndrome de enterocolitis inducida por proteínas alimentarias (Food Protein-Induced Enterocolitis Syndrome). Modificada de las referencias 35 y 36 .

De otro lado, en los pacientes con procesos no mediados por la IgE, las indicaciones para remitirlos al especialista, quien considerará la realización de una endoscopia se pueden observar en la Tabla 5.

\section{Hallazgos endoscópicos sugestivos de APLV}

Los hallazgos endoscópicos son inespecíficos y en diversas ocasiones no ayudan al diagnóstico de la proctocolitis inducida por la APLV. Entre los hallazgos endoscópicos sugestivos de alergia, principalmente cuando se sospecha de una esofagitis eosinofílica, se incluyen surcos lineales
(33-48 \%), anillos circulares (44-55\%), alteración del patrón vascular (41\%), pápulas blanquecinas $(27 \%)$, estenosis parciales (10-39\%) y estenosis completas (9\%).

La endoscopia puede ser normal en el 7-32 \% de los casos. Aunque los hallazgos endoscópicos sean normales, se deben tomar biopsias proximales y distales del esófago; de antro y cuerpo en el estómago, el duodeno y el bulbo, y en el colon de íleon, ciego y todos los cuadrantes para efectuar el estudio de gastroenteropatía eosinofílica, enfermedad celíaca, presencia de Helicobacter pylori, entre otras (32-34).

Tabla 5. Indicaciones de remisión al especialista en procesos no mediados por la $\operatorname{Ig} E^{*}$

\begin{tabular}{|c|c|}
\hline $\begin{array}{l}\text { Proctosigmoiditis } \\
\text { alérgica }\end{array}$ & $\begin{array}{l}\text { Sangrado rectal persistente, acompañado de } \\
\text { moco, dolor anal, fisuras anales, plicomas anales } \\
\text { (acrocordón), diarrea persistente, anemia, falla } \\
\text { en el crecimiento. }\end{array}$ \\
\hline $\begin{array}{l}\text { Es } \\
\text { eo }\end{array}$ & $\begin{array}{l}\text { Hiporexia, ingesta de abundante líquido con las } \\
\text { comidas, ingesta y masticación lentas, disfagia } \\
\text { o sensación de atoramiento, sospecha de } \\
\text { estenosis esofágica, falla en el crecimiento. }\end{array}$ \\
\hline $\begin{array}{l}\text { Gastroduodenitis } \\
\text { eosinofílica }\end{array}$ & $\begin{array}{l}\text { Dolor abdominal persistente, distensión } \\
\text { abdominal, vómito, hematemesis, signos } \\
\text { de malabsorción, anemia o deficiencias } \\
\text { nutricionales, falla en el crecimiento. }\end{array}$ \\
\hline $\begin{array}{l}\text { Colitis } \\
\text { eosinofílica }\end{array}$ & $\begin{array}{l}\text { Diarrea persistente, dolor y distensión abdominal, } \\
\text { sangrado digestivo bajo. }\end{array}$ \\
\hline
\end{tabular}

*Esta tabla es un resultado de lo acordado durante la reunión de expertos.

\section{Recomendaciones}

- Se sugiere que los procedimientos endoscópicos sean realizados por gastroenterólogos pediatras experimentados.

- Se recomienda la toma de biopsias de esófago, estómago, bulbo, duodeno, íleon, ciego y los cuadrantes del colon, y que se solicite un recuento de eosinófilos por campo y la clasificación de Marsh para la enfermedad celíaca.

\section{MANEJO NUTRICIONAL}

\section{Conceptos básicos}

El manejo nutricional del paciente debe ser individualizado y se requiere de un equipo multidisciplinario que incluya a un profesional en nutrición experto en el abordaje de la APLV.

De acuerdo con las recomendaciones de las guías de acción contra la alergia a la leche de vaca (Diagnosis and Rationale for Action against Cow's Milk Allergy, DRACMA) es necesario considerar los siguientes aspectos (14):

- Se debe realizar una estricta vigilancia nutricional durante el proceso. 
- Se recomienda una dieta de eliminación de mínimo 6 meses ( $<1$ año).

- La dieta de eliminación debe ser estricta, efectiva y completa. Se requiere evitar la inhalación y el contacto de la piel con productos o trazas que contengan PLV.

- Todas las dietas de eliminación deben ser nutricionalmente seguras. Es necesario evitar restricciones de macro y micronutrientes. Nunca se deben restringir otros tipos de alimentos si el diagnóstico es de APLV (huevo y pescado, entre otros).

\section{Objetivos del manejo nutricional}

El objetivo del manejo nutricional en los pacientes con APLV es mantener un adecuado estado nutricional, a fin de garantizar el crecimiento y el desarrollo del niño $(37,38)$.

\section{Fórmulas}

A continuación, se describen algunos factores que deben considerarse a la hora de elegir una fórmula para el manejo nutricional de los pacientes con $\operatorname{APLV}(37,39)$ (Tabla 6).

\section{Fórmulas hipoalergénicas}

Estas cumplen el criterio definido de $90 \%$ de tolerancia clínica (intervalo de confianza [IC], $95 \%$ ) en niños con alergia comprobada a la PLV. Se incluyen en las fórmulas hipoaler-
Tabla 6. Factores que se deben considerar para la elección de las fórmulas especiales

Malnutrición, presencia de anemia, hipoalbuminemia.

Riesgo de anafilaxia.

Edad del paciente.

Adherencia a la restricción dietética.

Modificada de las referencias 37 y 39

génicas las fórmulas extensamente hidrolizadas $(\mathrm{FEH})$ y las fórmulas de aminoácidos libres (FAA) (Tabla 7).

\section{Reto o desafío terapéutico}

- Se recomienda realizar el primer reto/desafío entre los primeros 6 meses y 1 año después del diagnóstico, en pacientes con síntomas leves.

- En caso de anafilaxia, el reto/desafío se realiza, como mínimo, 1 año después del episodio, bajo monitorización y vigilancia, preferiblemente en un centro hospitalario. Dicho reto lo determina el especialista competente.

- No es conveniente realizar el reto/desafío al mismo tiempo que se inicia la alimentación complementaria en el niño ( 6 meses de edad), pues si se presentan síntomas no se puede definir si son por causa de la alimentación complementaria o del reto/desafío.

Tabla 7. Fórmulas hipoalergénicas

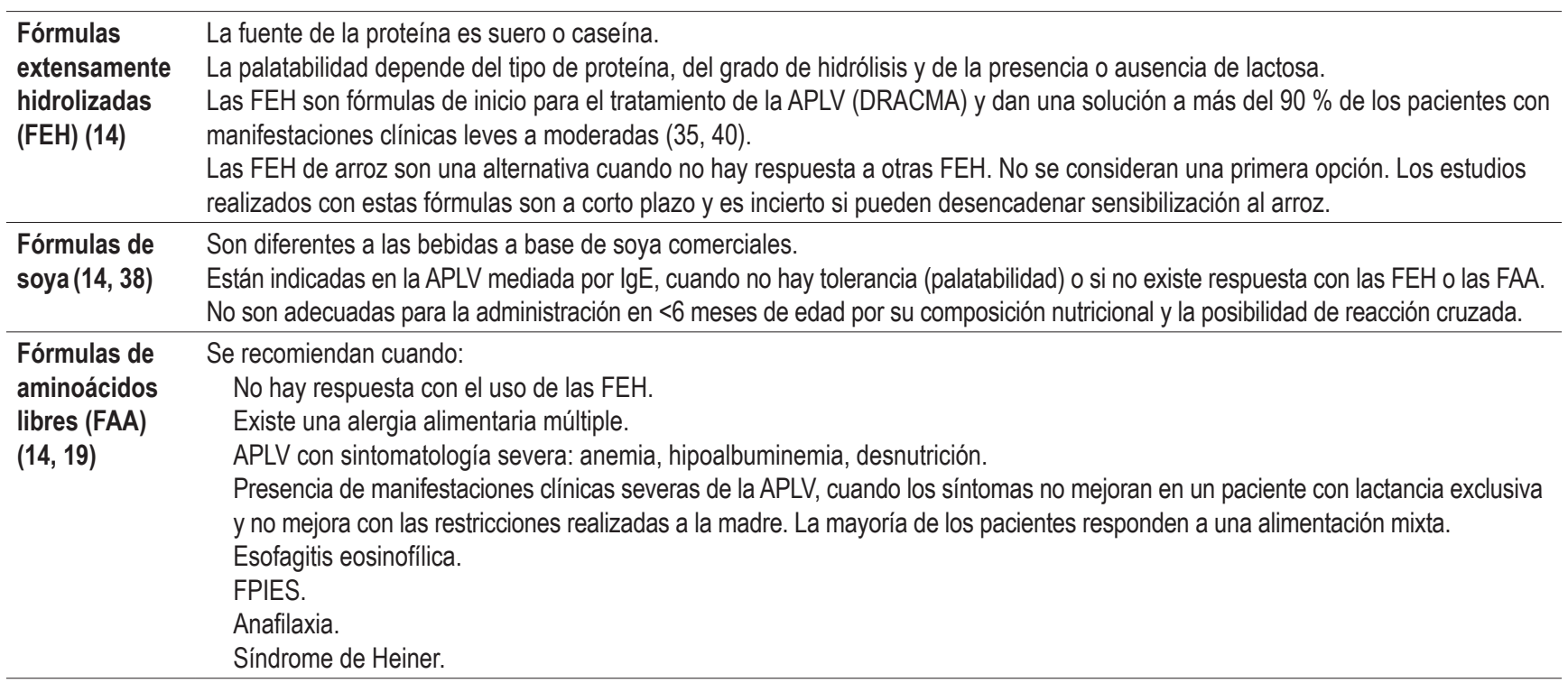

APLV: alergia a la proteína de la leche de vaca; DRACMA: acción contra la alergia a la leche de vaca (Diagnosis and Rationale for Action against Cow's Milk Allergy); IgE: inmunoglobulina E; FPIES: síndrome de enterocolitis inducida por proteínas alimentarias (Food Protein-Induced Enterocolitis Syndrome) 


\section{Recomendaciones en el embarazo y la lactancia materna exclusiva}

- No existe evidencia del beneficio en la restricción de alérgenos a la madre durante el embarazo, como métodos de prevención de la aparición de una APLV. Se debe evitar la restricción alimentaria a la madre durante la gestación y apuntar a una dieta adecuada, variada y balanceada que mantenga el peso recomendado.

- La lactancia exclusiva se asocia a múltiples beneficios y no se recomienda restringir la PLV a las madres como una forma de prevención. La lactancia es ideal de forma exclusiva hasta los 6 meses de edad, y de forma complementaria hasta los 24 meses o hasta que la madre o el hijo lo decidan.
- En pacientes con APLV, se requiere la estricta eliminación de la PLV de la dieta de la madre y del lactante. Se indica a la madre una dieta de eliminación de toda fuente de proteína láctea y se deben dar suplementos de calcio. Asimismo, se requiere informar a los padres y los cuidadores sobre las vías de sensibilización del niño y la revisión cuidadosa de las etiquetas de los productos que puedan tener componentes de proteína láctea (p. ej. cosméticos, jabones, cremas, lociones, pañitos húmedos, salsas, entre otros) (Tabla 8).

- Se recomienda motivar a las madres para continuar con la lactancia materna. Las manifestaciones gastrointestinales mejoran a las 2 semanas y las dermatológicas, a las 6 semanas de iniciar la restricción. Estas manifesta-

Tabla 8. Recomendaciones sobre las restricciones en el consumo de alimentos

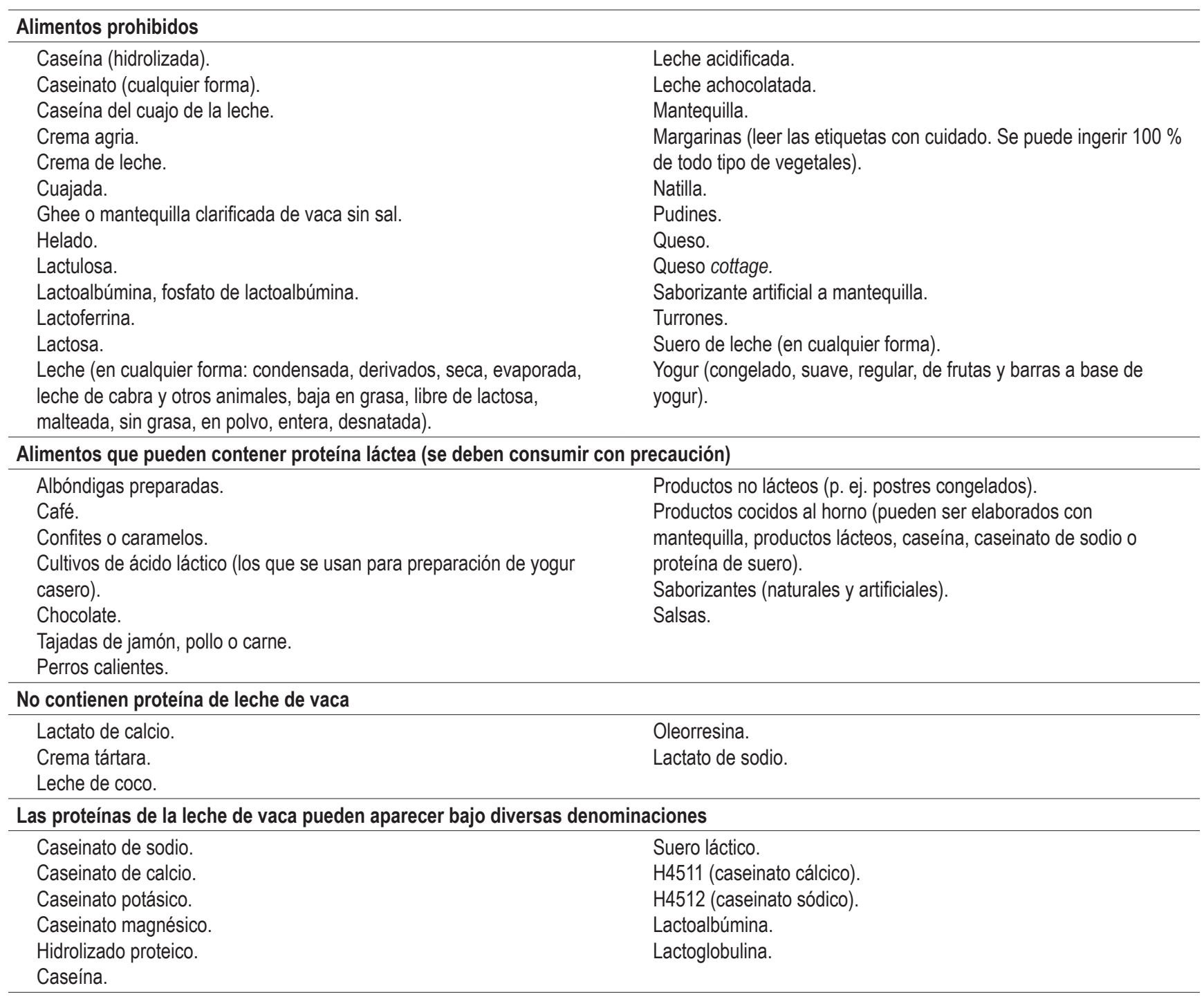

Modificada de las referencias 42-46. 
ciones no constituyen una razón para retirar la lactancia materna. Si no hay mejoría, la madre se debe remitir al especialista antes de suspender la lactancia materna o restringir otros.

- La alimentación complementaria se puede introducir entre los 4 y 6 meses de edad. Hay mayor riesgo de alergia si se introducen alimentos sólidos antes de los 4 meses.

- No existe evidencia de que retrasar la introducción de alimentos considerados alergénicos (p. ej. huevo, fresas), antes del primer año, reduzca el riesgo de alergia (población general y atópica) (41).

- No hay evidencia clara de que las fórmulas parcialmente hidrolizadas y las FEH prevengan algún tipo de alergia ni que sean mejores que la leche materna.

\section{Recomendaciones en niños que no reciben leche materna}

- Las fórmulas recomendadas en APLV son las FEH y las FAA.

- Los niños requieren de suplementación con micronutrientes como el calcio (cuando no se puede garantizar un volumen mínimo de $500 \mathrm{~mL}$ de leche materna o de fórmula), el hierro (en sangrado importante o en los pacientes con ferropenia) y la vitamina $\mathrm{D}$ (si se demuestra su deficiencia).

- Si la APLV persiste después del primer año, se requiere el uso de fórmulas especiales diseñadas para esa edad.

- No existe evidencia en Colombia de que las fórmulas llamadas hipoalergénicas (HA) o Confort sirvan para tratar a los pacientes con APLV o para la prevención de esta.

- No se recomienda el uso de bebidas elaboradas a base de almendras, arroz, coco y soya, entre otras, dada la alta probabilidad de reacción cruzada y debido a un aporte nutricional insuficiente.

\section{Recomendaciones adicionales}

- Prohibir los postres con mantequilla, leche, caseína o suero de leche (42-46) (Tabla 8).

- Leer la etiqueta de los postres con frutas.

- Prohibir los alimentos gratinados, cremosos, empanados y fritos.

- No es seguro el uso de leche de équidos o caprinos como alternativas.

\section{MANEJO FARMACOLÓGICO E INMUNOTERAPIA}

No existe un tratamiento farmacológico específico para el manejo de la APLV. Sin embargo, en los casos de alergia severa mediada por IgE, como la anafilaxia, la adrenalina es el tratamiento de elección.

La vida media de la adrenalina es corta y pueden requerirse varias dosis. En Colombia, su uso es intrahospitalario (no hay autoinyectores). Así, ante la presencia de una reacción anafiláctica se recomienda asistir inmediatamente al servicio de urgencias. Para el manejo de la anafilaxia con adrenalina intrahospitalaria, la dosis recomendada es de $0,15 \mathrm{mg}$ intramuscular, sin diluir, en $<25 \mathrm{~kg}$, con jeringa de aguja recambiable 21 corta en tercio medio y lateral de muslo. Repetir la dosis en 10-15 min si no hay respuesta. En $>25 \mathrm{~kg}$, la dosis es de 0,3 mg (guía de anafilaxia de la Academia Europea de Alergia e Inmunología Clínica [European Academy of Allergy and Clinical Immunology, EAACI ] $)(47,48)$.

En la última década, se ha investigado sobre otras terapias alternativas para el manejo de la APLV. Entre estas opciones se incluyen la inmunoterapia alérgeno específica, la terapia biológica, la medicina china y el uso de probióticos o prebióticos.

\section{Inmunoterapia}

$\mathrm{Su}$ objetivo es desensibilizar al paciente, o sea, aumentar el umbral de reactividad a un alérgeno y generar una tolerancia sostenida durante meses o años sin tratamiento adicional. Sin embargo, esta meta no siempre se alcanza y lo deseable es que no haya una reacción severa ante una exposición accidental (49-51) (Tabla 9). El tratamiento implica que el paciente esté recibiendo permanentemente dosis del alérgeno. Los primeros estudios de inmunoterapia en alergia alimentaria se hicieron en alergia al maní. La eficacia global de este tratamiento es del $35 \%$.

En síntesis, la inmunoterapia oral es más efectiva, pero presenta mayores eventos adversos, mientras que la epicutánea es menos efectiva, aunque se asocia a una menor aparición de eventos adversos.

\section{Terapia biológica}

El omalizumab, el ligelizumab y el quilizumab son terapias biológicas que se encuentran en investigación para el tratamiento de la APLV, principalmente de tipo IgE, y como coadyuvantes de la inmunoterapia $(52,53)$.

\section{Medicina tradicional china}

Las fórmulas herbales para el tratamiento de alergias alimentarias (Food Allergy Herbal Formula, [FAHF]) 1 y 2 consisten en una mezcla de hierbas chinas, cuyos ingredientes activos son la berberina y la limonina. Hasta el momento son tratamientos experimentales. En consecuencia, no se recomiendan. 
Tabla 9. Tipos de inmunoterapia.

\begin{tabular}{|c|c|}
\hline $\begin{array}{l}\text { Inmunoterapia } \\
\text { subcutánea }\end{array}$ & $\begin{array}{l}\text { No se utiliza en APLV, dados los riesgos que } \\
\text { implica. }\end{array}$ \\
\hline $\begin{array}{l}\text { Inmunoterapia } \\
\text { sublingual (extracto }\end{array}$ & $\begin{array}{l}\text { Solo se utiliza para investigación en alergia } \\
\text { alimentaria. }\end{array}$ \\
\hline $\begin{array}{l}\text { liquido del alérgeno } \\
\text { sublingual) }\end{array}$ & $\begin{array}{l}\text { En APLV: primer reporte de Boissieu (2006), } \\
70 \% \text { de desensibilización. Al parecer es más } \\
\text { segura. }\end{array}$ \\
\hline Inmunoterapia oral & $\begin{array}{l}\text { Se expone el paciente al alérgeno, a dosis } \\
\text { escalonadas por vía oral. } \\
\text { No está aprobada por la FDA en el tratamiento } \\
\text { de alergia alimentaria. } \\
\text { Efectos adversos frecuentes ( } 20 \% \text { ). } \\
\text { Las guías españolas de inmunoterapia oral la } \\
\text { recomiendan para el tratamiento de alergias a } \\
\text { la leche y el huevo. }\end{array}$ \\
\hline $\begin{array}{l}\text { Inmunoterapia } \\
\text { epicutánea }\end{array}$ & $\begin{array}{l}\text { En estudio. } \\
\text { Aplicación de PLV en forma de parches. } \\
\text { Las reacciones en la piel son frecuentes. } \\
\text { No hay reacciones graves. }\end{array}$ \\
\hline
\end{tabular}

APLV: alergia a la proteína de la leche de vaca; PLV: proteína de la leche de vaca; FDA: Administración de Medicamentos y Alimentos (Food and Drug Administration). Modificada de las referencias 49-51

\section{Probióticos y prebióticos}

No existe aún evidencia concluyente de que los probióticos y los prebióticos sean eficaces en el tratamiento de la APLV o en la prevención durante el último trimestre de embarazo y los primeros meses. Los datos sugieren que suplementar las fórmulas hidrolizadas con ciertas cepas de probióticos puede tener beneficios en términos de tolerancia oral, principalmente en el eccema atópico. Sin embargo, se requieren investigaciones adicionales para definir la especie, la dosis y los tiempos de terapia. La guía de 2016 de la Organización Mundial de Alergia (World Allergy Organization, WAO) sugiere el uso de probióticos, exclusivamente, en niños no lactados $(40,54)$.

\section{Terapia génica}

Aún se encuentra en fase de investigación y no existen terapias aprobadas.

\section{Recomendaciones}

- La dieta de eliminación sigue siendo el tratamiento de elección para la APLV.

- La inmunoterapia es el tratamiento más prometedor en un futuro mediato.

- Otras terapias coadyuvantes parecen mejorar la eficacia de la inmunoterapia y disminuir los efectos adversos, sin que exista aún evidencia suficiente para hacer una recomendación.

\section{Fuente de apoyo financiero}

Agradecemos a Danone Baby Nutrition Colombia, por su apoyo para realizar las reuniones. Esta compañía no tuvo injerencia en las discusiones, decisiones, ni en los conceptos académicos emitidos.

\section{REFERENCIAS}

1. Montijo-Barrios E, López-Ugalde MV, Ramírez-Mayans J, Anaya-Flórez MS, Arredondo-García JL, AzevedoTenorio I, et al. [Guía latinoamericana para el diagnóstico y tratamiento de alergia a las proteínas de la leche de vaca (GL-APLV)]. Rev Invest Clin. 2014;66 Suppl 2:S9-S72.

2. Koletzko S, Niggemann B, Arato A, Dias JA, Heuschkel R, Husby $S$, et al. Diagnostic approach and management of cow's-milk protein allergy in infants and children: ESPGHAN GI Committee practical guidelines. J Pediatr Gastroenterol Nutr. 2012;55(2):221-9. https://doi. org/10.1097/MPG.0b013e31825c9482

3. Schoemaker AA, Sprikkelman AB, Grimshaw KE, Roberts G, Grabenhenrich L, Rosenfeld L, et al. Incidence and natural history of challenge-proven cow's milk allergy in European children--EuroPrevall birth cohort. Allergy. 2015;70(8):963-72. https://doi.org/10.1111/all.12630

4. Boyce JA, Assaad A, Burks AW, Jones SM, Sampson HA, Wood RA, et al. Guidelines for the diagnosis and management of food allergy in the United States: summary of the NIAIDsponsored expert panel report. Nutr Res. 2011;31(1):61-75. https://doi.org/10.1016/j.nutres.2011.01.001

5. Rona RJ, Keil T, Summers C, Gislason D, Zuidmeer L, Sodergren E, et al. The prevalence of food allergy: a metaanalysis. J Allergy Clin Immunol. 2007;120(3):638-46. https://doi.org/10.1016/j.jaci.2007.05.026

6. Ávila MDL. Pérez J, Del Río BE, Rosas MA, Lerma L, Sienra JJL. Hipersensibilidad por prueba cutánea a alimentos en pacientes alérgicos en el Hospital Infantil de México Federico Gómez. Rev Alergia Mex 2002;49(3):74-79.

7. Martínez J, Méndez C, Talesnik E, Campos E, Viviani P, Sánchez D. Pruebas cutáneas de hipersensibilidad inmediata en una población pediátrica seleccionada. Rev Med Chile. 2005;133(2):195-201. https://doi.org/10.4067/ S0034-98872005000200007

8. Marrugo J, Hernández L, Villalba V. Prevalence of selfreported food allerg in Cartagena (Colombia) population. Allergol Immunopathol (Madr). 2008;36(6):320-4. https://doi.org/10.1016/S0301-0546(08)75863-4

9. Acevedo N, Sánchez J, Zakzuk J, Bornacelly A, Quiróz C, Alvarez Á, et al. Particular characteristics of allergic symptoms in tropical environments: follow up to 24 months in the FRAAT birth cohort study. BMC Pulm Med. 2012;12:13. https://doi.org/10.1186/1471-2466-12-13 
10. Solé D, Mallol J, Wandalsen GF, Aguirre V; Latin American ISAAC Phase 3 Study Group. Prevalence of symptoms of eczema in Latin America: results of the International Study of Asthma and Allergies in Childhood (ISAAC) Phase 3. J Investig Allergol Clin Immunol. 2010;20(4):311-23.

11. Arévalo M, Reyes MA, Victoria L, Villegas A, Badiel M, Herrera S. Asma y rinitis alérgica en pre-escolares en Cali. Colomb Med. 2003;34(1):4-8.

12. Dennis RJ, Caraballo L, García E, Rojas MX, Rondón MA, Pérez A, et al. Prevalence of asthma and other allergic conditions in Colombia 2009-2010: a cross-sectional study. BMC Pulm Med. 2012;12(17);1-9. https://doi. org/10.1186/1471-2466-12-17

13. Orsi M, Fernández A, Follett FR, Marchisone S, Saieg G, Busoni VB, et al. Alergia a la proteína de la leche de vaca. Propuesta de Guía para el manejo de los niños con alergia a la proteína de la leche de vaca. Arch Argent Pediatr. 2009; 107(5):459-470.

14. Fiocchi A, Brozek J, Schünemann H, Bahna SL, von Berg A, Beyer K, et al. World Allergy Organization (WAO) Diagnosis and Rationale for Action against Cow's Milk Allergy (DRACMA) Guidelines. Pediatr Allergy Immunol. 2010;21 Suppl 21:1-125. https://doi.org/10.1111/j.13993038.2010.01068.x

15. Björkstén B. Genetic and environmental risk factors for the development of food allergy. Curr Opin Allergy Clin Immunol. 2005;5(3):249-53. https://doi.org/10.1097/01. all.0000168790.82206.17

16. Eggesbø M, Botten G, Stigum H, Samuelsen SO, Brunekreef B, Magnus P. Cesarean delivery and cow milk allergy/ intolerance. Allergy. 2005;60(9):1172-3. https://doi. org/10.1111/j.1398-9995.2005.00857.x

17. Sánchez-Valverde F, Gil F, Martínez D, Fernández B, Aznal E, Oscoz M, et al. The impact of caesarean delivery and type of feeding on cow's milk allergy in infants and subsequent development of allergic march in childhood. Allergy. 2009;64(6):884-9. https://doi.org/10.1111/j.13989995.2008.01931.x

18. Vera JF, Ramírez A. Síntomas digestivos y respuesta clínica en lactantes con alergia a la proteína de leche de vaca. Rev Chil Pediatr. 2013;84:(6):641-649. https://doi. org/10.4067/S0370-41062013000600007

19. Luyt D, Ball H, Makwana N, Green MR, Bravin K, Nasser SM, et al. BSACI guideline for the diagnosis and management of cow's milk allergy. Clin Exp Allergy. 2014;44(5):642-72. https://doi.org/10.1111/cea.12302

20. Zuluaga LC, Ramírez R, Mejía LK, Vera JF. Outcomes of treating infants with cow's milk protein allergies with an extensively hydrolyzed serum-based formula. Rev Colomb Gastroenterol. 2018;33(2):111-115. https://doi. org/10.22516/25007440.253

21. Ministerio de Salud de Chile. Guía clínica: alergia a proteína de lecha de vaca. Santiago: Ministerio de Salud; 2012.

22. Pumphrey RS, Gowland MH. Further fatal allergic reactions to food in the United Kingdom, 1999-2006. J Allergy Clin
Immunol. 2007;119(4):1018-9. https://doi.org/10.1016/j. jaci.2007.01.021

23. Simons FE, Ardusso LR, Bilò MB,El-MagalYM,LedfordDK, Ring J, et al. World Allergy Organization anaphylaxis guidelines: summary. J Allergy Clin Immunol. 2011;127(3):587593. https://doi.org/10.1016/j.jaci.2011.01.038

24. Skripak JM, Matsui EC, Mudd K, Wood RA. The natural history of IgE-mediated cow's milk allergy. J Allergy Clin Immunol. 2007;120(5):1172-7.https://doi.org/10.1016/j. jaci.2007.08.023

25. Muraro A, Roberts G, Clark A, Eigenmann PA, Halken S, Lack G, et al. The management of anaphylaxis in childhood: position paper of the European academy of allergology and clinical immunology. Allergy. 2007;62(8):857-71. https:// doi.org/10.1111/j.1398-9995.2007.01421.x

26. Sampson HA, Muñoz-Furlong A, Campbell RL, Adkinson NF, Bock SA, Branum A, et al. Second symposium on the definition and management of anaphylaxis: summary report-Second National Institute of Allergy and Infectious Disease/Food Allergy and Anaphylaxis Network symposium. J Allergy Clin Immunol. 2006;117(2):391-7. https:// doi.org/10.1016/j.jaci.2005.12.1303

27. Braganza SC, Acworth JP, Mckinnon DR, Peake JE, Brown AF. Paediatric emergency department anaphylaxis: different patterns from adults. Arch Dis Child. 2006;91(2):159-163. https://doi.org/10.1136/adc.2004.069914

28. Latcham F, Merino F, Lang A, Garvey J, Thomson MA, Walker-Smith JA, et al. A consistent pattern of minor immunodeficiency and subtle enteropathy in children with multiple food allergy. J Pediatr. 2003;143(1):39-47. https://doi. org/10.1016/S0022-3476(03)00193-8

29. Butt AM, Murch SH, Ng CL, Kitching P, Montgomery S, Philips A, et al. Upregulated eotaxin expression and T cell infiltration in the basal and papillary epithelium in cows' milk associated reflux oesophagitis. Arch Dis Child. 2002;87(2):124130. https://doi.org/10.1136/adc.87.2.124

30. Berni Canani R, Nocerino R, Terrin G, Coruzzo A, Cosenza L, Leone L, et al. Effect of Lactobacillus GG on tolerance acquisition in infants with cow's milk allergy: a randomized trial. J Allergy Clin Immunol. 2012;129(2):580-2,582.e1-5. https://doi.org/10.1016/j.jaci.2011.10.004

31. Sicherer SH, Sampson HA. Food allergy: A review and update on epidemiology, pathogenesis, diagnosis, prevention, and management. J Allergy Clin Immunol. 2018;141(1):41-58. https://doi.org/10.1016/j.jaci.2017.11.003

32. Kim HP, Vance RB, Shaheen NJ, Dellon ES. The prevalence and diagnostic utility of endoscopic features of eosinophilic esophagitis: a meta-analysis. Clin Gastroenterol Hepatol. 2012;10(9):988-96.e5. https://doi.org/10.1016/j. cgh.2012.04.019

33. Pasha SF, DiBaise JK, Kim HJ, De Petris G, Crowell MD, Fleischer DE, et al. Patient characteristics, clinical, endoscopic, and histologic findings in adult eosinophilic esophagitis: a case series and systematic review of the medical literature. Dis Esophagus. 2007;20(4):311-9. https://doi. org/10.1111/j.1442-2050.2007.00721.x 
34. Liacouras CA, Spergel JM, Ruchelli E, Verma R, Mascarenhas M, Semeao E, et al. Eosinophilic esophagitis: a 10-year experience in 381 children. Clin Gastroenterol Hepatol. 2005;3(12):1198-206. https://doi.org/10.1016/ S1542-3565(05)00885-2

35. Lee KM, Lim HC, Kim JH, Yoon YH, Park HJ, Lee SI. Clinical implications of endoscopically suspected eosinophilic esophagitis. Korean J Gastroenterol. 2010;56(5):28592. https://doi.org/10.4166/kjg.2010.56.5.285

36. Moawad FJ, Schoepfer AM, Safroneeva E, Ally MR, Chen YJ, Maydonovitch CL, et al. Eosinophilic oesophagitis and proton pump inhibitor-responsive oesophageal eosinophilia have similar clinical, endoscopic and histological findings. Aliment Pharmacol Ther. 2014;39(6):603-8. https://doi. org/10.1111/apt.12636

37. Uppal V, Kreiger P, Kutsch E. Eosinophilic Gastroenteritis and Colitis: a Comprehensive Review. Clin Rev Allergy Immunol. 2016;50(2):175-88. https://doi.org/10.1007/ s12016-015-8489-4

38. Fleischer DM, Spergel JM, Assa'ad AH, Pongracic JA. Primary prevention of allergic disease through nutritional interventions. J Allergy Clin Immunol Pract. 2013; 1(1):2936. https://doi.org/10.1016/j.jaip.2012.09.003

39. Giovannini M, D’Auria E, Caffarelli C, Verduci E, Barberi S, Indinnimeo L, et al. Nutritional management and follow up of infants and children with food allergy: Italian Society of Pediatric Nutrition/Italian Society of Pediatric Allergy and Immunology Task Force Position Statement. Ital J Pediatr. 2014;40:1. https://doi.org/10.1186/1824-7288-40-1

40. Fiocchi A, Dahda L, Dupont C, Campoy C, Fierro V, Nieto A. Cow's milk allergy: towards an update of DRACMA guidelines. World Allergy Organ J. 2016;9(1):35. https://doi. org/10.1186/s40413-016-0125-0

41. Fewtrell M, Bronsky J, Campoy C, Domellöf M, Embleton N, Fidler Mis N, et al. Complementary Feeding: A Position Paper by the European Society for Paediatric Gastroenterology, Hepatology, and Nutrition (ESPGHAN) Committee on Nutrition. J Pediatr Gastroenterol Nutr. 2017;64(1):119-132. https://doi.org/10.1097/MPG.0000000000001454

42. Molnár K, Pintér P, Győrffy H, Cseh A, Müller KE, Arató A, et al. Characteristics of allergic colitis in breast-fed infants in the absence of cow's milk allergy. World J Gastroenterol. 2013;19(24):3824-30. https://doi.org/10.3748/wjg.v19. i24.3824

43. Yu MC, Tsai CL, Yang YJ, Yang SS, Wang LH, Lee CT, et al. Allergic colitis in infants related to cow's milk: clinical characteristics, pathologic changes, and immunologic findings. Pediatr Neonatol. 2013;54(1):49-55. https://doi. org/10.1016/j.pedneo.2012.11.006
44. Kokkonen J, Karttunen TJ. Lymphonodular hyperplasia on the mucosa of the lower gastrointestinal tract in children: an indication of enhanced immune response? J Pediatr Gastroenterol Nutr. 2002;34(1):42-6. https://doi. org/10.1097/00005176-200201000-00010

45. Hwang JB, Park MH, Kang YN, Kim SP, Suh SI, Kam S. Advanced criteria for clinicopathological diagnosis of food protein-induced proctocolitis. J Korean Med Sci. 2007;22(2):213-217. http://dx.doi.org/10.3346/ jkms.2007.22.2.213

46. Mansueto P, Iacono G, Seidita A, D’Alcamo A, Sprini D, Carroccio A. Review article: intestinal lymphoid nodular hyperplasia in children-the relationship to food hypersensitivity. Aliment Pharmacol Ther. 2012;35(9):1000-9. https://doi.org/10.1111/j.1365-2036.2012.05062.x

47. European Academy of Allergy and Clinical Immunology (EAACI). Food Allergy and Anaphylaxis Guidelines. Zúrich: EAACI; 2014.

48. Jones SM, Burks AW. Food Allergy. N Engl J Med. 2017;377(12):1168-1176. https://doi.org/10.1056/ NEJMcp 1611971

49. Ebisawa M, Ito K, Fujisawa T; Committee for Japanese Pediatric Guideline for Food Allergy, The Japanese Society of Pediatric Allergy and Clinical Immunology, The Japanese Society of Allergology. Japanese guidelines for food allergy 2017. Allergol Int. 2017;66(2):248-264. https://doi. org/10.1016/j.alit.2017.02.001

50. Martorell A, Alonso E, Echeverría L, Escudero C, GarcíaRodríguez $\mathrm{R}$, Blasco $\mathrm{C}$, et al. Oral Immunotherapy for Food Allergy: A Spanish Guideline. Immunotherapy Egg and Milk Spanish Guide (ITEMS Guide). Part I: Cow Milk and Egg Oral Immunotherapy: Introduction, Methodology, Rationale, Current State, Indications, Contraindications, and Oral Immunotherapy Build-up Phase. J Investig Allergol Clin Immunol. 2017;27(4):225-237. https://doi. org/10.18176/jiaci.0177

51. Kobernick AK, Burks AW. Active treatment for food allergy. Allergol Int. 2016;65(4):388-395. https://doi. org/10.1016/j.alit.2016.08.002

52. MacGinnite A. Update on Potential Therapies for IgEMediated Food Allergy. Curr Allergy Asthma Rep. 2017;17(1):4. https://doi.org/10.1007/s11882-017-0671-8

53. Hamad A, Burks WA. Emerging Approaches to Food Desensitization in Children. Curr Allergy Asthma Rep. 2017;17(5):32. https://doi.org/10.1007/s11882-017-0700-7

54. Vandenplas Y. Prevention and Management of Cow's Milk Allergy in Non-Exclusively Breastfed Infants. Nutrients. 2017 Jul 10;9(7). pii: E731. https://doi.org/10.3390/ nu9070731 